\title{
Il trapianto di organi al tempo del COVID
}

\author{
Massimo Cardillo \\ Direttore Generale del Centro Nazionale Trapianti, Roma - Italy
}

La diffusione della pandemia da COVID-19 ha cambiato radicalmente la vita di tante persone in tutto il mondo e, oltre a provocare migliaia di vittime, ha sottoposto i sistemi sanitari a una pressione organizzativa senza precedenti, quantomeno nella storia recente. I professionisti sanitari, attivi sul territorio e negli ospedali, si sono trovati ad affrontare una vera emergenza, che ha coinvolto in prima battuta i reparti di terapia intensiva.

Soprattutto all'inizio, questa crisi così grave e inaspettata ha preoccupato in particolare chi, per una ragione o per l'altra, vive una fragilità che lo rende più vulnerabile ai rischi connessi all'infezione da COVID-19. Tra questi soggetti, ci sono i pazienti trapiantati in regime di immunosoppressione e le persone in lista d'attesa per un trapianto a causa di una grave insufficienza d'organo.

Proprio per questo, sin dalla segnalazione dei primi focolai di infezione nel nord del Paese, la rete trapiantologica italiana è stata allertata per mettere in atto misure che da un lato potessero garantire il proseguimento delle attività di donazione e trapianto e, dall'altro, fare in modo che i trapianti fossero eseguiti in sicurezza.

Il 2019 era stato un anno di incremento dell'attività (2.766 segnalazioni di potenziali donatori, 1.763 donatori effettivi, 3.813 trapianti: il secondo miglior anno di sempre per volumi complessivi, con le liste d'attesa in continua riduzione), con un buon aumento anche del numero dei trapianti da vivente, e l'effetto della pandemia rischiava di vanificare questi sforzi.

Mentre la curva dell'infezione continuava la sua ascesa esponenziale, si registrava un calo verticale dell'attività donativa e trapiantologica. I dati dei primi due mesi dell'anno avevano fatto registrare una media di 50 segnalazioni di donatori potenziali e di 90 trapianti alla settimana: il 15 marzo l'andamento settimanale era precipitato a 36 segnalazioni e a 41 trapianti soltanto. Come atteso, la saturazione dei livelli di occupazione dei posti letto nelle terapie intensive da parte

Received: March 24, 2021

Accepted: March 31, 2021

Published online: April 15, 2021

Indirizzo per la corrispondenza:

Massimo Cardillo

Centro Nazionale Trapianti

Istituto Superiore di Sanità

Viale Regina Elena, 299

00161 Roma - Italy

massimo.cardillo@iss.it dei pazienti affetti da COVID-19, che, in caso di decesso, non sono candidabili al prelievo di organi e tessuti a causa dell'infezione in atto, stava precludendo l'accesso alle rianimazioni alle altre tipologie di pazienti, il cui eventuale accertamento di morte encefalica avrebbe lasciato spazio alla possibilità di una donazione a beneficio di chi aspetta un trapianto.

L'impatto nei mesi di picco della prima ondata di pandemia è stato importante e ha fatto registrare in Italia un calo delle donazioni e dei trapianti vicino al $40 \%$, con una sospensione dei programmi in alcuni centri e un blocco generalizzato dei programmi di trapianto da donatore vivente. La rete italiana ha, però, retto bene, rispetto a quanto accaduto in altri Paesi, come Francia e Spagna, che hanno avuto, nello stesso periodo, riduzioni doppie.

Alcuni eventi di quel periodo sono stati emblematici per testimoniare la capacità del sistema trapianti nazionale di resistere alla pressione della pandemia e di continuare a curare i pazienti, basti pensare al trapianto di polmone eseguito il 21 marzo al Papa Giovanni XXIII di Bergamo, epicentro dell'infezione, e quello al Policlinico di Milano, in un ragazzo di 18 anni con un danno irreversibile dei polmoni, provocato proprio dal coronavirus. Sono state affrontate e spesso risolte anche criticità organizzative, come nel caso del trapianto di midollo eseguito al Bambino Gesù di Roma in un bimbo di 2 anni affetto da linfoistiocitosi emofagocitica primaria, con cellule staminali fatte arrivare dalla Turchia con volo dell'Aeronautica Militare in urgenza, che ha superato il blocco delle frontiere.

Davanti alla necessità di contenere il più possibile il calo delle donazioni e il suo effetto drammatico, la Rete Nazionale Trapianti ha attivato una strategia su tre livelli di destinazione: il primo istituzionale e di governance sanitaria, il secondo verso i pazienti e il terzo verso l'opinione pubblica. Tutte le misure intraprese sono state discusse e promosse grazie a un costante coinvolgimento della Consulta tecnica permanente per i trapianti, l'organo collegiale che opera a supporto del Centro Nazionale Trapianti, dei rappresentanti delle Regioni e della Direzione generale della prevenzione sanitaria del Ministero della Salute.

La prima misura è stata quella di segnalare alle Regioni la necessità di mantenere l'attività di prelievo e trapianto, in quanto livello essenziale di assistenza, urgente e non differibile, e in quanto connessa alla disponibilità di un donatore, condizione per definizione non programmabile. Nello stesso tempo, venivano definiti criteri di sicurezza per l'utilizzo dei 
donatori e per lo screening dei pazienti da avviare al trapianto e veniva attivato un sistema di monitoraggio dell'impatto dell'infezione sui pazienti in attesa e trapiantati, grazie all'integrazione del sistema informativo dei trapianti (SIT) con i dati della piattaforma COVID dell'Istituto Superiore di Sanità (ISS).

Per quanto riguarda il secondo livello, con il coinvolgimento dei coordinamenti regionali, dei centri trapianto locali e delle associazioni, tra le quali ANED ha svolto un ruolo determinante, sono stati attivati servizi di supporto diretto ai pazienti trapiantati e in lista d'attesa, con l'obiettivo di rispondere ai dubbi più ricorrenti e alle necessità contingenti di una fascia della popolazione oggettivamente più esposta ai rischi connessi alla pandemia. Esempi di questa attività sono la pagina riepilogativa sul sito istituzionale www.trapianti. salute.gov.it di tutte le iniziative intraprese per il contrasto alla pandemia e il webinar dedicato ai pazienti e alle associazioni, realizzato nel mese di maggio.

Infine, c'è stato il terzo livello di azione, quello destinato alla popolazione generale, attraverso i canali di informazione: la struttura di comunicazione istituzionale e di ufficio stampa del CNT si è adoperata per trovare una chiave d'accesso efficace per veicolare messaggi comprensibili ed efficaci, sistematizzando il flusso di informazioni provenienti dalla rete e canalizzandoli in funzione delle esigenze dei media e della rete stessa.

Nel frattempo, era importante monitorare l'impatto dell'infezione nei pazienti: a questo scopo, il CNT ha analizzato i dati risultanti dall'incrocio dei database SIT e della piattaforma COVID dell'ISS. È emerso che, sebbene i pazienti trapiantati o in attesa di trapianto abbiano un rischio di infezione con Sars-CoV-2 più alto della popolazione generale, a causa delle terapie immunosoppressive alle quali sono sottoposti e, nel caso dei pazienti in dialisi, della frequenza di contatti nelle strutture ospedaliere, il rischio sembra, comunque, maggiore per i pazienti in attesa di trapianto rispetto a chi lo ha già ricevuto. Un risultato che ci ha incoraggiato a proseguire con le normali attività dei centri trapianto, adottando le adeguate misure di sicurezza per i pazienti.

Nello stesso studio, è stato possibile mettere in correlazione il rischio di infezione con alcuni fattori genetici che possono influenzare la capacità di combattere l'infezione stessa: il gruppo sanguigno e una variante genica del principale sistema che regola la risposta immune nell'uomo, il sistema di istocompatibilità, denominato HLA. Questo sistema codifica per la produzione di alcune particolari proteine, chiamate antigeni HLA, responsabili del funzionamento del nostro sistema immunitario e della risposta di rigetto al trapianto, ma anche del riconoscimento e della risposta agli agenti infettivi, come, per esempio, virus o batteri.

Riguardo al gruppo sanguigno, i soggetti di gruppo A sembrano avere una probabilità lievemente maggiore di ammalarsi, mentre le persone con gruppo 0 sarebbero più resistenti all'infezione. Parlando, invece, degli antigeni HLA, è la variante HLA-DRB ${ }^{*} 08$ quella più frequentemente associata sia ai casi di positività che a una prognosi peggiore di malattia.

Se confermati con studi più ampi, questi risultati potrebbero rivelarsi importanti non solo per indirizzare le scelte nella gestione clinica dei pazienti più a rischio, ma anche come fattori con cui orientare le campagne di vaccinazione, per dare la precedenza alle popolazioni più suscettibili.

Nelle settimane della seconda ondata della pandemia, rispetto al calo dell'attività di donazione e trapianto, osservato nei mesi di picco della prima ondata, la rete è riuscita a mantenere un volume di prestazioni in linea con quello degli ultimi mesi, grazie anche all'attenzione e alle misure che le Regioni e gli ospedali hanno dedicato al mantenimento di questa attività, nonostante le difficoltà organizzative legate alla gestione della pandemia da COVID-19.

II Centro Nazionale Trapianti, insieme al Ministero della Salute, è impegnato in un costante monitoraggio delle attività della rete trapiantologica e in una puntuale revisione delle situazioni più critiche, al fine di fornire tutto il supporto necessario affinché i tanti pazienti che aspettano un organo possano continuare a ricevere in tutta sicurezza quel livello essenziale di assistenza che è il trapianto.

\section{Disclosures}

Conflict of interest: The author declares no conflict of interest. Financial support: This article received no specific grant from any funding agency in the public, commercial, or not-for-profit sectors. 